\title{
An Equivalent-Circuit Topology for Lossy Non-Symmetric Reciprocal Two-Ports
}

\author{
Alberto Hernández-Escobar, Elena Abdo-Sánchez, Member, IEEE, Pablo Mateos-Ruiz, Jaime Esteban, \\ Teresa M. Martín-Guerrero, Member, IEEE, and Carlos Camacho-Peñalosa, Life Senior Member, IEEE
}

\begin{abstract}
An equivalent-circuit topology for two-port lossy non-symmetric reciprocal networks is proposed. The circuit topology is based on the eigenstate decomposition. The proposed circuit consists of two immittances and two transformers with a single complex turns ratio, all obtained from the eigenvalues and eigenvectors of the admittance or impedance matrix of the network in a straightforward way. The interconnection of the circuit elements is simple and compact, and the real parts of its immittances are always positive. To verify its behavior, the equivalent circuit of two different leaky-wave antenna unit cells is extracted: a series-fed coupled patch radiating element, and the complementary strip-slot. Simulated and measured data are provided for the study cases, highlighting the satisfactory behavior of the equivalent circuit over a very broad bandwidth. A simple asymmetry model is also proposed that can be used advantageously in the analysis and design of non-symmetric twoports, like unit cells of leaky-wave antennas.
\end{abstract}

Index Terms-Asymmetry, eigenstates, equivalent circuits, lattice network, leaky-wave antenna, open stopband

\section{INTRODUCTION}

$\mathbf{T}$ HERE is a growing interest in the modeling of lossy nonsymmetric reciprocal two-port electromagnetic structures by means of equivalent circuits. This interest has been fostered, to a great extent, by periodic Leaky-Wave Antennas (LWAs), since it has been shown that the introduction of certain asymmetries in their unit cell can mitigate the so-called open-stopband effect at broadside [1], [2]. The performance of the LWA is indeed governed by the asymmetries of the unit cell and their control can lead to improved LWA design strategies and behavior. Having a proper equivalent circuit can, in general, help provide some physical insight into the involved electromagnetic phenomena [3], and can, in the particular case of LWAs, be crucial for extracting the propagation constant that determines the performance of such antennas [4].

Manuscript received Month DD, YYYY; revised Month DD, YYYY; accepted Month DD, YYYY.

A. Hernández-Escobar, E. Abdo-Sánchez, P. Mateos-Ruiz, T. M. MartínGuerrero and C. Camacho-Peñalosa are with the Instituto Universitario de Investigación en Telecomunicación, Escuela Técnica Superior de Ingeniería de Telecomunicación, Universidad de Málaga, Andalucía Tech, 29010 Málaga, Spain (e-mail: ahe@ic.uma.es).

J. Esteban is with the Information Processing and Telecommunications Center, Escuela Técnica Superior de Ingenieros de Telecomunicación, Universidad Politécnica de Madrid, 28040 Madrid, Spain.

This work was supported by the Spanish Ministerio de Educación, Cultura y Deporte (Programa para la Formación del Profesorado Universitario) under Grant FPU15/06457 and by the Spanish Ministerio de Ciencia, Innovación y Universidades (MCIU), the Agencia Estatal de Investigación (AEI) and the Fondo Europeo de Desarrollo Regional (FEDER) (Programa Estatal de I+D+i Orientada a los Retos de la Sociedad) under grant RTI2018-097098-J-I00.
From a purely mathematical point of view, three complex parameters are necessary for the complete specification of a dissipative reciprocal two-port structure, so the equivalent circuit must contain at least three lossy circuit elements or their equivalents [5]. Examples of general equivalent circuits like these are the $\Pi$ or $\mathrm{T}$ networks found in the classic literature. A given equivalent circuit is characterized by its constituent elements or components (mainly immittances, transformers, and transmission line sections or reference plane shifts) and its topology (the way the elements are interconnected). In the case of non-dissipative two-ports there is a wide variety of available equivalent circuits (see, for instance, [5], [6]). However, there is not such a variety of choices for the case of dissipative reciprocal two-ports.

One of the problems with dissipative reciprocal two-ports is that, depending on the topology of the equivalent circuit, immitances with negative real parts may appear, which is not a desirable characteristic, although it does not compromise energy conservation in the two-port as a whole. This modeling problem (the appearance of negative real parts in the immittances of the equivalent circuits) was already addressed in the early days of microwave technology. For instance, Felsen and Oliner [7] further elaborated on an original idea from Weissfloch [8] and proposed to separate the dissipative equivalent circuit into lossy and lossless portions. In doing so, they obtained what they called canonical networks or equivalent circuits composed by two transmission line sections (or two reference plane shifts), one reactance or susceptance, one ideal transformer with real turns ratio, and two nonnegative resistors (a total of six elements, although only two of them are lossy). The idea of separating the dissipative twoport into lossy and lossless portions has reached our days and, very recently, Zappelli [9] has proposed equivalents circuits for dissipative reciprocal two-port devices based on this separation approach. These equivalent circuits contain non-negative resistors and, in its simpler form (compact configuration), has seven (real) elements and an intricate extraction procedure.

In the case of dissipative symmetric (certainly reciprocal) two-ports, the lattice network can be considered as the optimum equivalent circuit topology, since it guarantees the realizability of the two immittances required to model the response of any realizable symmetric network. Moreover, the lattice network provides a profound physical insight into the circuit behavior, since any response can be considered as a linear combination of the two eigenstates (even- and oddmode excitations) of the structure. Because of this, in recent years, the spotlight has been put on the quest for lossy non- 
symmetric equivalent circuits that mimic, to some extent, the properties of the lattice network. Unfortunately, equivalent circuits based on the lattice-network topology require the structure to be symmetric, and the derivation of an extension of this topology for non-symmetric circuits has proved not to be straightforward.

In an attempt to elaborate on some design methodology to choose the degree of asymmetry needed to cancel the broadside effect in LWAs, an equivalent circuit based on the symmetric lattice network was proposed in [2]. Although this equivalent circuit was useful for the purpose of [2], this circuit lacked the main aforementioned properties of the lattice networks which might limit its application to other problems. An alternative to this circuit is the equivalent circuit topology proposed in [10]. The latter follows an eigenstate formulation approach [11] and, unlike the circuit in [2], it preserves the property of symmetric lattice networks of being decomposable in eigenstates. This property of decomposition has been satisfactorily exploited very recently in [12] to propose the design flow of a modified microstrip Franklin unit cell for its use in LWAs.

Although the benefits of the equivalent circuit topology proposed in [10] have been already proved in specific LWA designs [12], this model has revealed to have two main problems. The first one is that the real parts of their immittances can be negative unless power orthogonality between the eigenstates is enforced by adding a frequency-dependent shift in the reference planes. This is an undesirable behavior for an equivalent circuit of a passive network. The second problem is that, when the two ports are isolated ( $y_{12}$ or $z_{12}$ is zero or close to zero), the equivalent circuit is not able to properly degenerate into this limit case.

A novel equivalent circuit topology for non-symmetric structures is proposed here, which overcomes the main drawbacks of the equivalent circuits proposed in [2], [9], [10]. The proposed circuit topology consists of two immittances and two transformers with a single complex turns ratio. This results in three complex parameters, the minimum number of parameters required unlike [9]. Even more, the extraction procedure is absolutely straightforward, with simple explicit equations for all the circuit elements. The circuit is based on the eigenstate decomposition proposed in [10], thus, it is able to properly separate the even-like and odd-like modes in a very broad bandwidth, providing more physical insight than [2]. Apparently, the difference of the proposed topology with respect to [10] can seem not very significant; however, these modifications have a dramatic effect on the immittances: their values are the eigenvalues of the immittance matrix of the structure. As an important consequence of this, the real parts of these two immittances are always positive (regardless of the choice of reference planes). Additionally, the circuit is able to gracefully degenerate into the limit case defined by $y_{12}=0$ or $z_{12}=0$, which broadens its applicability and usefulness. Obviously, the topology degenerates into the wellknown lattice network in the case of symmetric two-ports. The performance evaluation of the equivalent circuit is addressed by extracting its element values for two LWA unit cells: one based on the full-wave electromagnetic simulation of a series- fed coupled patch, and the other based on the measurements of a complementary strip-slot. These performance evaluations include an analysis of the influence on the equivalent circuit elements of the two-port degree of asymmetry. In addition, a model is found for the behavior of the two-ports as a function of the degree of asymmetry using the proposed equivalent circuit topology. A simple methodology is proposed to design these LWA unit cells. Unlike the methodologies proposed in [2], [12], this one is simple and general.

This paper is structured as follows. Section II shows the eigenstate decomposition of a generic two-port. Section III proposes the new topology for both the admittance and impedance parameters. Section IV shows two examples of the application of the proposed equivalent circuit to model non-symmetric structures. Section V proposes a model for the asymmetry and shows its applicability to LWA design. Finally, Section VI summarizes the main conclusions.

\section{THEORETICAL BACKGROUND}

In [10], it was found that a non-symmetric reciprocal two-port network can be decomposed using an eigenstate formulation. For the sake of completeness, this argument is summarized here. Two decompositions were proposed, one of them using the admittance parameters and the other using the impedance parameters. From the admittance matrix of the network (the reasoning for the impedance matrix is analogous),

$$
[Y]=\left[\begin{array}{ll}
y_{11} & y_{12} \\
y_{12} & y_{22}
\end{array}\right]
$$

its eigenvalues can be obtained as

$$
\begin{aligned}
& \lambda_{1}=\frac{y_{11}+y_{22}+\sqrt{\left(y_{11}-y_{22}\right)^{2}+4 y_{12}^{2}}}{2} \\
& \lambda_{2}=\frac{y_{11}+y_{22}-\sqrt{\left(y_{11}-y_{22}\right)^{2}+4 y_{12}^{2}}}{2}
\end{aligned}
$$

and their associated normalized eigenvectors can be obtained as

$$
\begin{gathered}
\overrightarrow{v_{1}}=\frac{1}{\sqrt{|p|^{2}+1}}\left[\begin{array}{l}
p \\
1
\end{array}\right] \\
\overrightarrow{v_{2}}=\frac{1}{\sqrt{|p|^{2}+1}}\left[\begin{array}{c}
-1 \\
p
\end{array}\right],
\end{gathered}
$$

where

$$
p=\frac{y_{11}-y_{22}+\sqrt{\left(y_{11}-y_{22}\right)^{2}+4 y_{12}^{2}}}{2 y_{12}} .
$$

By the definition of the eigenvalues and eigenvectors, it is possible to write, then,

$$
\begin{aligned}
& {[Y] \overrightarrow{v_{1}}=\lambda_{1} \overrightarrow{v_{1}}} \\
& {[Y] \overrightarrow{v_{2}}=\lambda_{2} \overrightarrow{v_{2}}}
\end{aligned}
$$

and, by interpreting these expressions in terms of circuits, the eigenvector $\overrightarrow{v_{i}}$ can be seen as applied voltages in each of the ports that produce the current $\lambda_{i} \overrightarrow{v_{i}}(i=1,2)$. Unifying the expressions (5a) and (5b) and separating the result into the sum of two matrices, it can be written as

$$
[Y]\left[\begin{array}{ll}
\overrightarrow{v_{1}} & \overrightarrow{v_{2}}
\end{array}\right]=\left[\begin{array}{lll}
\lambda_{1} & \overrightarrow{v_{1}} & \overrightarrow{0}
\end{array}\right]+\left[\begin{array}{ll}
\overrightarrow{0} & \lambda_{2} \\
v_{2}
\end{array}\right]
$$


Then, it is possible to split the $[Y]$ matrix into two other admittance matrices, $\left[Y^{a}\right]$ and $\left[Y^{b}\right]$, as

$$
\begin{gathered}
{[Y]=\left[\begin{array}{ll}
\lambda_{1} \overrightarrow{v_{1}} & \overrightarrow{0}
\end{array}\right]\left[\begin{array}{ll}
\overrightarrow{v_{1}} & \overrightarrow{v_{2}}
\end{array}\right]^{-1}+\left[\begin{array}{ll}
\overrightarrow{0} & \lambda_{2} \overrightarrow{v_{2}}
\end{array}\right]\left[\begin{array}{ll}
\overrightarrow{v_{1}} & \overrightarrow{v_{2}}
\end{array}\right]^{-1}} \\
{[Y]=\left[Y^{a}\right]+\left[\begin{array}{l}
Y^{b} \\
{[Y}
\end{array}\right.}
\end{gathered}
$$

Their values are given by

$$
\begin{aligned}
& {\left[Y^{a}\right]=\lambda_{1}\left[\begin{array}{ll}
\frac{p^{2}}{p^{2}+1} & \frac{p}{p^{2}+1} \\
\frac{p}{p^{2}+1} & \frac{1}{p^{2}+1}
\end{array}\right]} \\
& {\left[Y^{b}\right]=\lambda_{2}\left[\begin{array}{ll}
\frac{1}{p^{2}+1} & \frac{-p}{p^{2}+1} \\
\frac{-p}{p^{2}+1} & \frac{p^{2}}{p^{2}+1}
\end{array}\right] .}
\end{aligned}
$$

Analogously, for the impedance formulation, the separation into submatrices is the following:

$$
\begin{gathered}
{[Z]=\left[Z^{a}\right]+\left[Z^{b}\right]} \\
{\left[Z^{a}\right]=\frac{1}{\lambda_{1}}\left[\begin{array}{ll}
\frac{p^{2}}{p^{2}+1} & \frac{p}{p^{2}+1} \\
\frac{p}{p^{2}+1} & \frac{1}{p^{2}+1}
\end{array}\right]} \\
{\left[Z^{b}\right]=\frac{1}{\lambda_{2}}\left[\begin{array}{ll}
\frac{1}{p^{2}+1} & \frac{-p}{p^{2}+1} \\
\frac{-p}{p^{2}+1} & \frac{p^{2}}{p^{2}+1}
\end{array}\right] .}
\end{gathered}
$$

Since the admittance matrix is the inverse matrix of the impedance matrix, by the properties of the eigenvalues, the impedance-matrix eigenvalues are the inverse of the admittance-matrix eigenvalues and their eigenvectors are the same. For this reason, in this derivation, the same eigenvalues of the immittance matrix, $\lambda_{1}$ and $\lambda_{2}$, and the same relation between the coefficient of their eigenvectors, $p$, have been used, regardless of whether the admittance or impedance matrix is considered.

Several circuit topologies for the subnetworks $\left[Y^{a}\right],\left[Y^{b}\right]$, $\left[Z^{a}\right]$ and $\left[Z^{b}\right]$ were proposed in [10]. However, they were the cause of its two main problems. The first one is that a frequency-dependent shift in the reference planes must be added to obtain positive real parts for the circuit immitances. The second problem is that when ports 1 and 2 are isolated from each other ( $y_{12}$ or $z_{12}$ are close to zero), none of the topologies in [10] is capable to reproduce simultaneously the input impedances at the two circuit ports.

\section{Proposed Equivalent Circuit}

A new circuit topology for the subnetworks is proposed which solves the aforementioned drawbacks. The key point is to place a complex-turns-ratio transformer between two identical admittances, as depicted inside the dashed rectangles of Fig. 1. This modification has a dramatic impact on the performance of the equivalent circuit.

A suitable analogous topology for the impedance matrix case is also proposed and it is shown inside the dashed rectangles of Fig. 2. Note that, in both cases, the equivalent circuit is defined by three complex parameters, namely $\lambda_{1}, \lambda_{2}$, and $p$. The extraction of these parameters over frequency is straightforward, since they are, respectively, the eigenvalues of the admittance matrix, given by (2a) and ( $2 b$ ), and the ratio between the components of its eigenvectors (4).

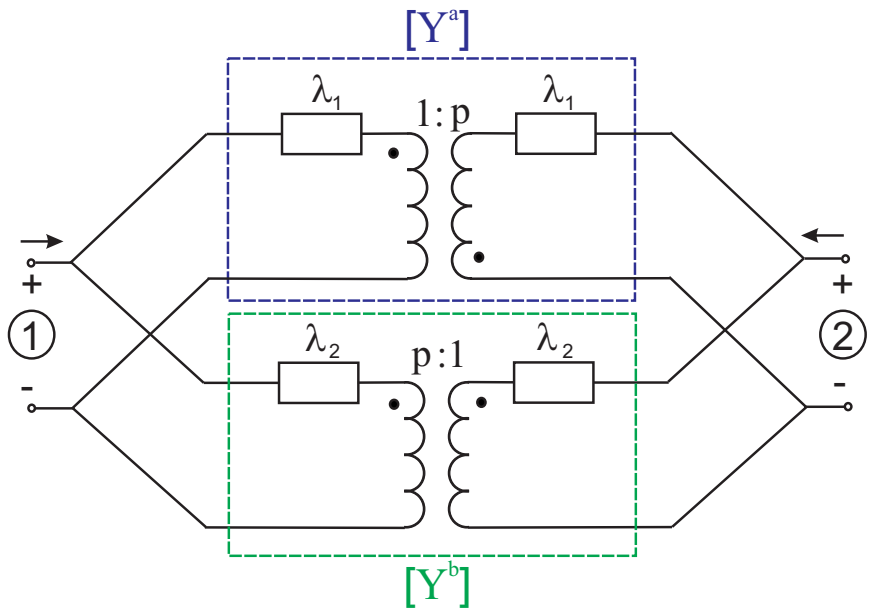

Fig. 1. Proposed eigenstate-based equivalent circuit using the admittance matrix.

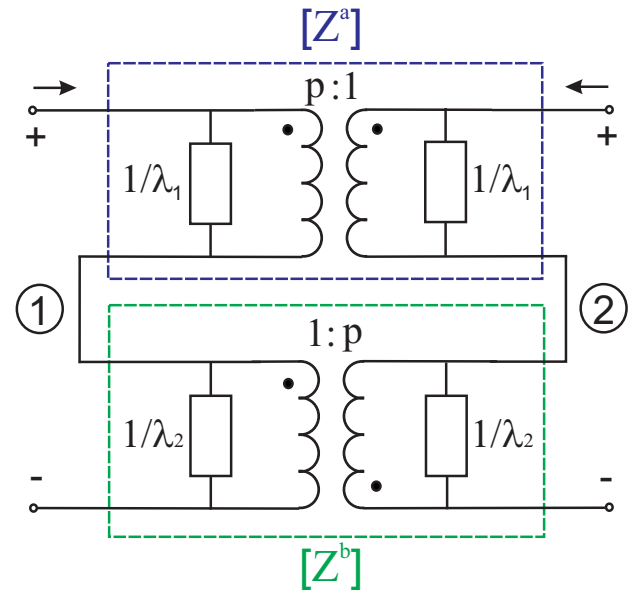

Fig. 2. Proposed eigenstate-based equivalent circuit using the impedance matrix.

A relevant characteristic of these topologies is that the values of the immittances are the same as the eigenvalues of the admittance/impedance matrices. As proved in the Appendix, the real parts of the eigenvalues of the admittance matrix of a lossy reciprocal network are always positive or zero. Thus, every admittance of the equivalent circuit will never have negative real parts, provided the two-port is passive and reciprocal. Regarding the impedance-matrix-based equivalent circuit, the real parts of its impedances are always positive, since the inverse of a complex number with a positive real part will also have a positive real part.

Note that the turns ratios of the transformers are defined by a single parameter $p$, which is, in general, a complex number. It is important to note that complex-turns-ratio transformers are elements used in the synthesis of networks [13]. It is also important to realize that these transformers are two-ports that, depending on the load conditions at their ports, can be lossy or active. If the turns ratio is real, the transformer is lossless. The role played by these two transformers in the equivalent circuit is clearly illustrated by computing the dissipated power 
in the two-port under arbitrary excitation. This power is given by

$$
\begin{aligned}
P_{d i s}=\frac{1}{2} & \operatorname{Re}\left\{V_{1}^{*} I_{1}+V_{2}^{*} I_{2}\right\}= \\
= & \frac{1}{2}|A|^{2} \operatorname{Re}\left\{\lambda_{1}\right\}+\frac{1}{2}|B|^{2} \operatorname{Re}\left\{\lambda_{2}\right\}+ \\
& +\frac{1}{2} \operatorname{Re}\left\{\frac{\left(p-p^{*}\right)\left(A^{*} B \lambda_{2}-A B^{*} \lambda_{1}\right)}{\left|p^{2}\right|+1}\right\},
\end{aligned}
$$

which leads to

$$
\begin{aligned}
P_{d i s}=|A|^{2} P_{d i s}^{a} & +|B|^{2} P_{\text {dis }}^{b}+ \\
& +\frac{\operatorname{Im}\{p\}}{\left|p^{2}\right|+1} \operatorname{Im}\left\{A B^{*} \lambda_{1}-A^{*} B \lambda_{2}\right\},
\end{aligned}
$$

where $A$ and $B$ are the complex amplitudes of the linear combination of eigenvectors that describe an arbitrary excitation, i.e.,

$$
\left[\begin{array}{l}
V_{1} \\
V_{2}
\end{array}\right]=\frac{1}{\sqrt{\left|p^{2}\right|+1}}\left\{A\left[\begin{array}{l}
p \\
1
\end{array}\right]+B\left[\begin{array}{c}
-1 \\
p
\end{array}\right]\right\} .
$$

The first and second terms of (11) are, respectively, the powers dissipated $\left(P_{d i s}^{a}\right.$ and $\left.P_{d i s}^{b}\right)$ in each subcircuit when only the corresponding eigenmode is excited. It is important to highlight that $P_{d i s}^{a}$ and $P_{d i s}^{b}$ are always positive or zero as $\operatorname{Re}\left\{\lambda_{1}\right\}$ and $\operatorname{Re}\left\{\lambda_{2}\right\}$ are non-negative. It is straightforward to prove that, when only one of the eigenmodes is excited, the voltages at both ports of the corresponding transformer are zero (virtual short-circuit) and, thus, the dissipated power in the transformer is zero, even if the turns ratio is complex. The third term of (11) is the so-called interaction term and accounts for the non-orthogonality of the eigenmodes. Note that this term is zero if the turns ratio is real, i.e., the eigenmodes are orthogonal. On the contrary, if the turns ratio is complex, the eigenmodes are non-orthogonal and the interaction term is different from zero (positive or negative). In this case the complex-turns-ratio transformers are active or lossy and provide the required power to account for the energy conservation. The physical origin of the non-orthogonality of the eigenmodes is the asymmetry of the two-port. However, the asymmetry of the circuit does not necessarily imply the non-orthogonality of the eigenmodes. In particular, if the twoport is either resistive or lossless, the resulting turns ratios are real, which implies that the eigenmodes are orthogonal although the two-port is asymmetric.

Two limit cases should be mentioned, because of the capacity of the proposed circuit to properly represent both situations. The first one is when $y_{12}=0\left(z_{12}=0\right)$ and the ports are isolated. The turn ratios of the transformers tend either to zero or infinity $(p=0$ or $p \rightarrow \infty)$ and therefore, the two transformers exhibit an open circuit and a short circuit in their ports, which isolates the two ports of both subnetworks. As a result, the input admittance seen at each port will be $\lambda_{1}$ and $\lambda_{2}$, which are the theoretical values of the input admittances of the isolated two-port. Given the value of $p$, the interaction term of (11) vanishes, which corresponds to the fact that there is no interaction between one port and the other.

The second limit case is the symmetric network, with $p=1$. The transformers then act either as a direct connection or as

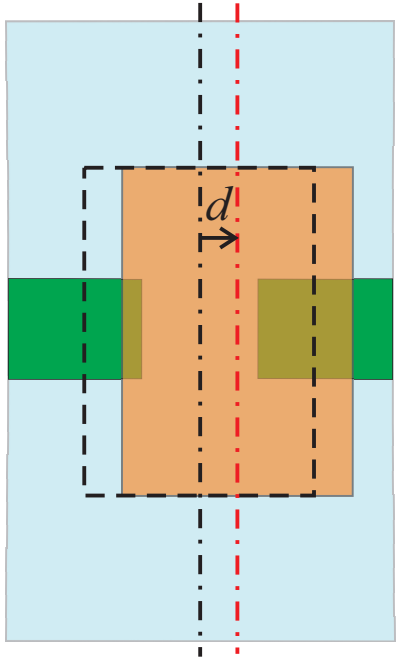

(a)

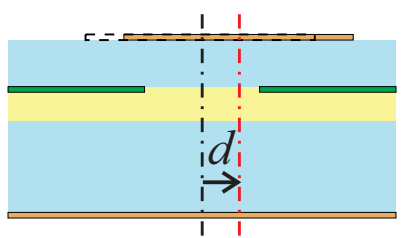

(b)

Fig. 3. The SFCP unit cell proposed in [2]. (a) Top view. (b) Side view.

a crossover, and the resulting circuit topology becomes the classic lattice network with series admittances of value $\lambda_{2}$ and cross admittances of value $\lambda_{1}$. Once again, the interaction term of (11) vanishes (in this case because $p$ is real). This is an expected result, since the modes of the lattice network are always orthogonal. Consequently, the lattice network becomes a particular case of the proposed equivalent circuit.

\section{CAse Studies}

In this section, the performance of the proposed equivalentcircuit topology is evaluated by extracting its component values for two, previously proposed, different unit cells of LWAs. The component values are extracted from the simulated or measured admittance parameters using the procedure specified in Section III. The addition of a suitable amount of asymmetry to these structures was already proposed in [2] and [14] to solve the problem of the open-stopband effect at broadside.

\section{A. Series-Fed Coupled Patch}

The first analyzed structure is the so-called Series-Fed Coupled Patch (SFCP) [2]. It consists of an on-top-stacked patch with gap coupling to the feeding line, as shown in Fig. 3. In [2], an equivalent circuit and its design methodology were proposed to identify the optimum level of asymmetry (in this case, moving the patch along the feeding line axis a distance $d$ ). The process, however, is elaborated, complex, and does not provide much in terms of physical insight.

With the aim of illustrating the advantages of the equivalentcircuit topology proposed in this work, several SFCP structures 


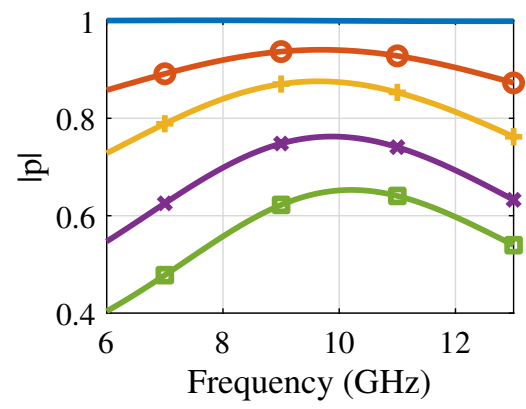

(a)

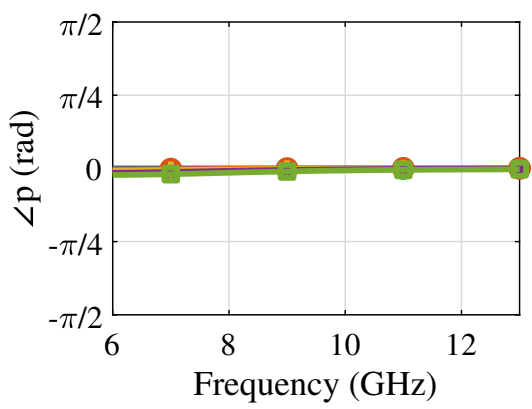

(d)

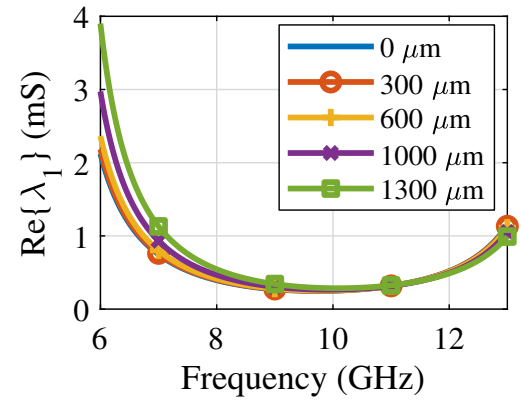

(b)

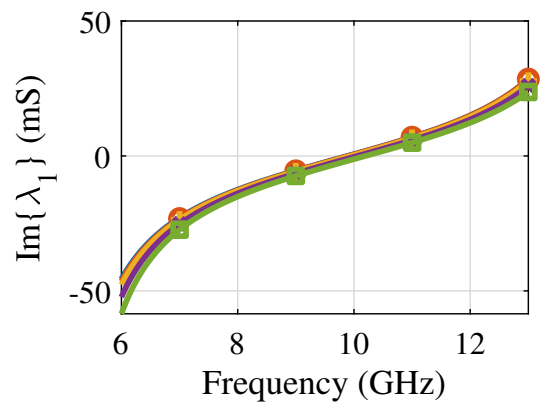

(e)

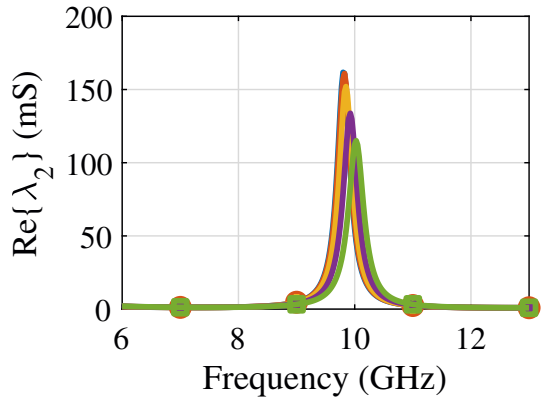

(c)

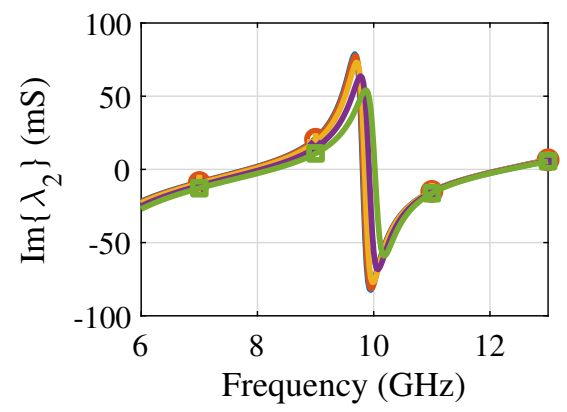

(f)

Fig. 4. Circuit parameters of the artificial SFCP unit cell for several asymmetries ( $d$ parameter) using the proposed equivalent circuit. (a) Magnitude of $p$. (b) Real part of $\lambda_{1}$. (c) Real part of $\lambda_{2}$. (d) Phase of $p$. (e) Imaginary part of $\lambda_{1}$. (f) Imaginary part of $\lambda_{2}$.

with the same dimensions as in [2], but with different degrees of asymmetry, $d$, were simulated using the commercial software HFSS. The eigenvalues and associated eigenvectors of the resulting admittance matrices were computed, which form the equivalent circuit from Fig. 1, and are shown in Fig. 4. One of the targets of this study is to check how the parameters change when increasing its degree of asymmetry. To have a reference for the degrees of asymmetry analyzed, if $d=1650 \mu \mathrm{m}$ the patch and microstrip would have no overlapping area in one of the sides, whereas $d=0$ corresponds to the symmetric case.

In all cases, even with the numerical errors expected from a simulation, the real parts of the admittances are positive, and the equivalent circuit behaves smoothly also at lower frequencies, where $y_{12}$ is low. Additionally, it can be seen that the turns ratios of the transformers are almost real, which means that the even-like and odd-like modes are also almost orthogonal, as explained in Section III. It is also noteworthy that the values of the immittances barely change when the level of asymmetry is changed. This is a very relevant result, since it means that the information about the asymmetry of the structure is mainly in the magnitude of the turns ratio of the transformers. From the physical point of view, this is not surprising as can be understood as the almost no alteration of the eigenmodes of the structure when a slight asymmetry is introduced. This asymmetry affects mainly and almost uniquely the interaction between the two eigenmodes, thus corroborating the powerful physical insight of the proposed topology.

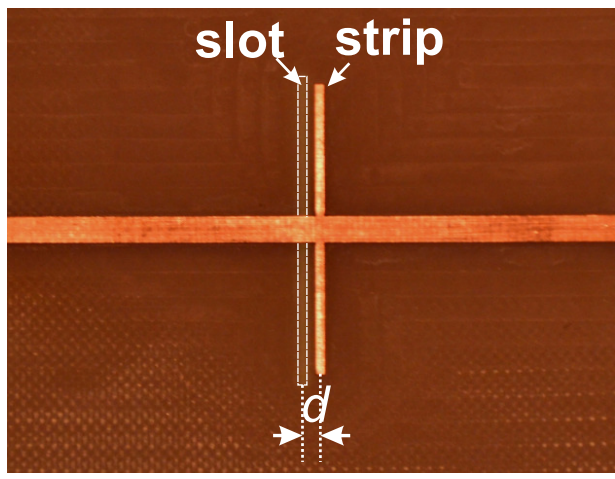

Fig. 5. Backlit photograph of a prototype of the complementary strip-slot with certain misalignment $d$.

\section{B. Complementary Strip-Slot}

In order to validate the new circuit topology with actual measurements, the so-called complementary strip-slot has been chosen as the second case study. This structure was proposed in [15] as a planar radiating element with outstanding broadband matching. It consists of a slot etched on the ground plane of a microstrip line. The complementary stub (strip) of the slot is placed on the microstrip layer, symmetrically aligned to the slot, as a matching element. This structure is especially interesting for the present analysis because the contribution of the strip is associated with the even mode, and that of the slot, with the odd mode. A consequence of the eigenstate separation property of the proposed equivalent circuit is that 
it can separate the contribution of these two modes and, thus, the contribution of the slot and stub, even if some asymmetry is added. This introduction of asymmetry to the structure was proposed in [14] to reduce the broadside open-stopband effect when building an LWA with this element.

Several prototypes of the strip-slot element with different degrees of misalignment $d$ have been fabricated. Fig. 5 shows a partially backlit photograph of one of them. This misalignment is introduced by moving the strip and the slot a distance $d / 2$ towards Port 2 and Port 1 , respectively. In this case, the element values of the equivalent circuit have been extracted from the measured S-parameters with respect to $Z_{0}=50 \Omega$, and the reference planes have been symmetrically placed $0.7 \mathrm{~mm}$ away from the center of the slot. To have a reference for the degrees of misalignment analyzed, the case of $d=540 \mu \mathrm{m}$ corresponds to the minimum value for which the strip and the slot have no overlapping area (they are adjacent elements loading the microstrip), whereas $d=0$ corresponds to the symmetric case. The lengths of the strip and the slot are such that their first resonant frequencies (total length equal to $\lambda / 2$ ) appear around $5.4 \mathrm{GHz}$. The structure is fabricated on GML 1032 substrate with $\epsilon_{r}=3.2$ and 30 mil thickness. The TRL calibration technique was used to obtain the measurements. The calibration kit consisted of three "Lines" to cover the whole band of interest. Some anomalies occurred at the frequencies where the calibration changed from one "Line" to another, with the largest errors between 9.4 and $9.8 \mathrm{GHz}$.

These structures were already analyzed using the previous eigenstate-based equivalent circuit from [10]. However, in order to obtain admittances with positive real parts, power orthogonality had to be enforced using frequency-dependent shifts in the reference planes. Furthermore, when these different reference planes were used, the variation of the admittances with the asymmetry was higher. The equivalent circuit of these measured structures using the topology proposed in this work is shown in Fig. 6. With this topology, the real parts of the admittances are positive in the measured frequency range, but between $9.4 \mathrm{GHz}$ and $9.8 \mathrm{GHz}$. At these frequencies, the condition (16c) from the Appendix is not fulfilled as a consequence of the anomalies introduced by the calibration kit. As in the case of the SFCP, the variation of the admittances with the asymmetry is small, even though they are different physical structures. Thus, the $\lambda_{1}$ and $\lambda_{2}$ values still represent properly the slot and the strip admittances, separately, with the transformation ratio of the transformer absorbing the effect of the asymmetry. With this case study, the eigenstate-separation capabilities of the equivalent circuit are illustrated, in addition to its robustness in the presence of errors and inaccuracies from measurements.

\section{ASymMetry Model AND ITS USE IN LWA UNIT-CELL} DESIGN

It is evident that, at least for some structures, the $p$ parameter seems to contain all or most of the information about the asymmetry. Assuming that, it has been found out that it is possible to model $p$ as:

$$
p=1-g(f) d^{n}
$$

where $d$ is the misalignment of the different layers of the structure causing the asymmetry, $n$ is a frequency-independent positive real value, and $g(f)$ is a complex function of the frequency. Function $g(f)$ and exponent $n$ must be found for each structure from the data of two or more different asymmetry values. Once these function and exponent are obtained, this simple model allows predicting the parameter $p$ of the circuit for other asymmetries and, since the eigenvalues are assumed invariant with the asymmetry level, the behavior of the complete structure can be predicted. Note that this model will be only valid for values of $d$ that are not too high.

The model of the $p$ parameter of the SFCP case has been computed. From the $300 \mu \mathrm{m}$ and the $600 \mu \mathrm{m}$ cases, the mean of the function $g(f)$ and parameter $n$ have been found. The first one can be seen in Fig. 7, and the value found for the exponent is $n=1$. The same values are obtained if other two different asymmetry cases are used, which shows the consistency of the model. To prove that (13) properly models the $p$ parameter, the computed values using the model and the original simulated values are shown in Fig. 8, where more asymmetry cases ranging from 150 and $600 \mu \mathrm{m}$ were included. It can be seen that the model can both interpolate and extrapolate results. However, it was not able to predict cases with more asymmetry than $600 \mu \mathrm{m}$ as accurately. Also, it is especially noteworthy the fact that the same dependence with frequency of $g(f)$ is valid for different asymmetry values over such a wide bandwidth.

Additionally, the model of the complementary strip-slot case has been also computed using the results of Fig. 6 from the $150 \mu \mathrm{m}$ and the $270 \mu \mathrm{m}$ misalignment values. The function $g(f)$ can be seen in Fig. 9, and the value of $n$ is 0.45 . The values of $p$ predicted by the model and the measured values are shown in Fig. 10. Using the values of $p$ given by the model, the eigenvalues for any single case and equations (7b), (8a), and $(8 \mathrm{~b})$, it is possible to predict the admittance parameters of the structure with other asymmetry levels. To show this concept, the magnitude and phase of the $S$ parameters of the measured cases are plotted in Fig. 11 along with those obtained from the modeled $p$ and the eigenvalues of the symmetric case. It can be seen that the predicted and measured values are very similar in a very wide range of frequencies, even though each measurement is from a different manufactured board. This further proves that, once the asymmetry model is obtained, it is possible to get the two-port parameters of the structure for other asymmetry levels without more simulations or measurements.

Furthermore, the advantages of the new circuit topology are used to give a design example of the previously analyzed SFCP unit cell. LWAs suffer from the open stop-band effect at broadside, which deteriorates the radiation performance at this angle. One of the approaches to mitigate this undesirable effect is to minimize the reflection of the unit cell at the broadside frequency, since, at that frequency, the reflections of each element are added in phase, leading to a strong mismatching and a drop in the radiation efficiency [16]. The asymmetry 


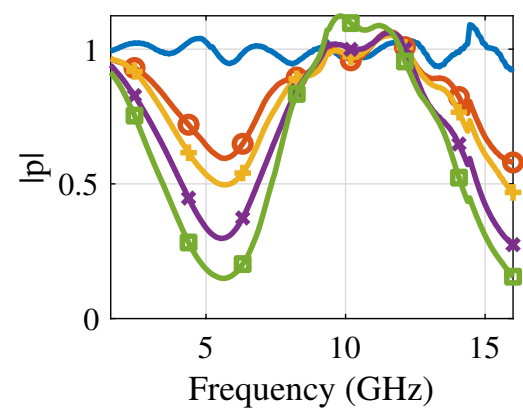

(a)

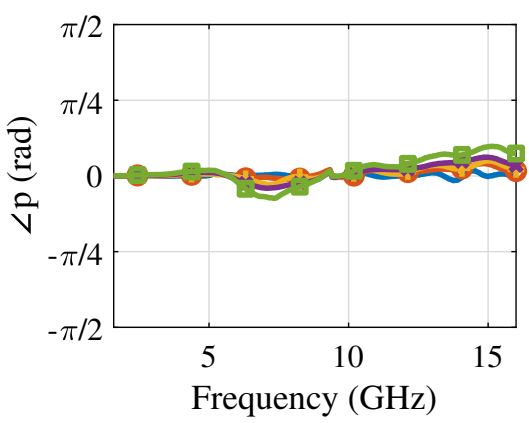

(d)

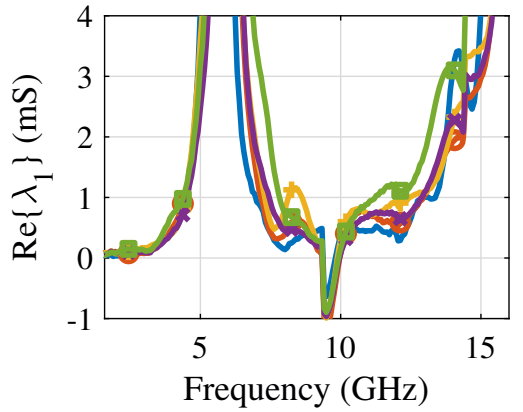

(b)

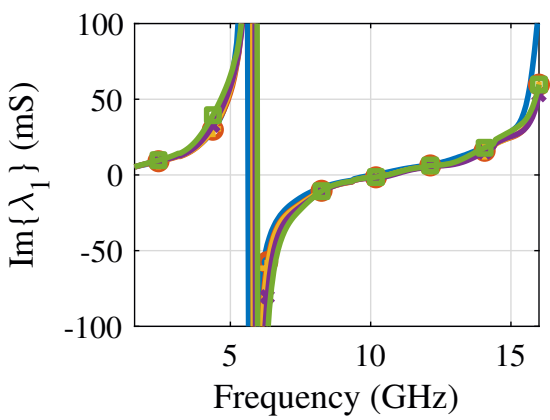

(e)

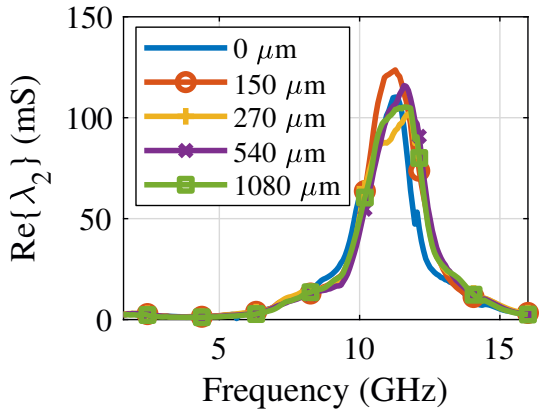

(c)

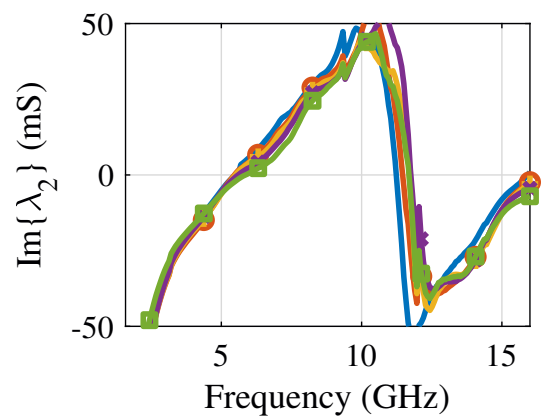

(f)

Fig. 6. Circuit parameters of the measured strip-slot unit cells for several asymmetries ( $d$ parameter) using the proposed equivalent circuit. (a) Magnitude of $1 / p$. (b) Real part of $\lambda_{1}$. (c) Real part of $\lambda_{2}$. (d) Phase of $1 / p$. (e) Imaginary part of $\lambda_{1}$. (f) Imaginary part of $\lambda_{2}$.

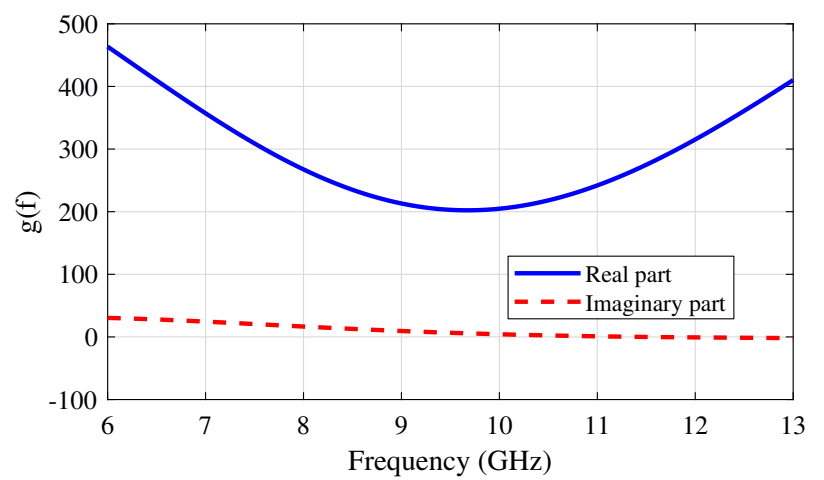

Fig. 7. $g(f)$ function of the asymmetry model for the SFCP unit cell.

level of this unit cell was already designed in [2] to eliminate the open-stopband effect following this approach, however, a more general, different design procedure is illustrated here using the proposed asymmetry model. The goal is to find the best asymmetry level, the parameter $d$, to minimize the reflection at broadside by changing its Bloch impedance at its first port, $Z_{B+}$, without changing the radiation properties of the unit cell.

The proposed procedure is as follows. First, it is assumed (supported by the results from Section IV) that all the information about the asymmetry of the structures are absorbed by the turns ratio of the transformers $(p)$ and, thus, the immittances $\left(\lambda_{1}\right.$ and $\lambda_{2}$ ) do not change (significantly) with the asymmetry level $(d)$. It is possible, then, to find the dependence of the parameter $p$ with $d$ at the broadside frequency, $f_{0}$, using (13). Afterwards, the value of the Bloch admittance at the first port of the cell, $1 / Z_{B+}$, as a function of $d$ is calculated as following:

$$
Y_{B+}=\frac{p^{2}-1}{p^{2}+1} \frac{\lambda_{1}-\lambda_{2}}{2} \pm \sqrt{\left(\frac{\lambda_{1}+\lambda_{2}}{2}\right)^{2}-p^{2}\left(\frac{\lambda_{1}-\lambda_{2}}{p^{2}+1}\right)^{2}} .
$$

From this expression, the optimum value of $d$ can be found so the reflection coefficient is minimum at $f_{0}$.

The procedure has been applied to the SFCP unit cell. Using (14), the magnitude of the predicted reflection coefficient in function of $d$ is shown in Fig. 12, where it is possible to see that the minimum value is obtained when $d=220 \mu \mathrm{m}$. To verify the result, the simulation results of the same coefficient for the different levels of asymmetry are shown in Fig. 13, where it is possible to appreciate that the lowest coefficient is obtained when $d=220 \mu \mathrm{m}$, which coincides with the result from Fig. 12.

\section{CONCLUSION}

In this paper, a general eigenstate-based equivalent circuit topology for lossy non-symmetric reciprocal two-ports is proposed. The circuit has only three complex parameters, namely two lossy immittances and the complex turns ratio of the transformers. It has been rigorously proved that the proposed circuit topology guarantees immittances with non-negative real parts. Moreover, the proposed equivalent circuit can efficiently 


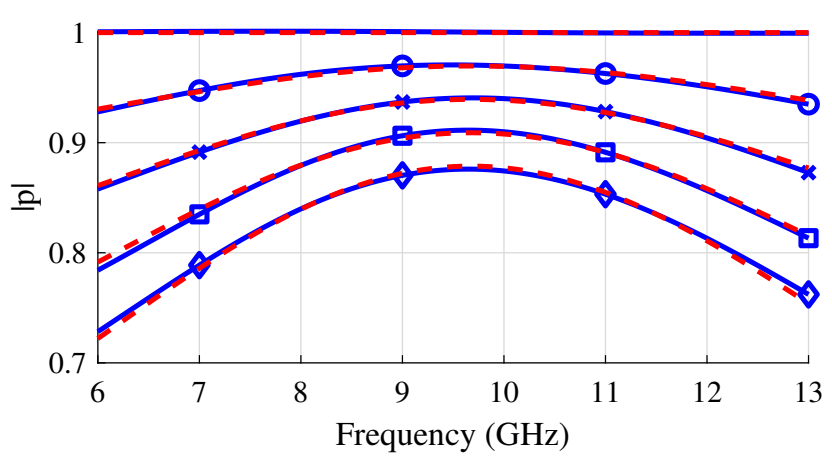

(a)

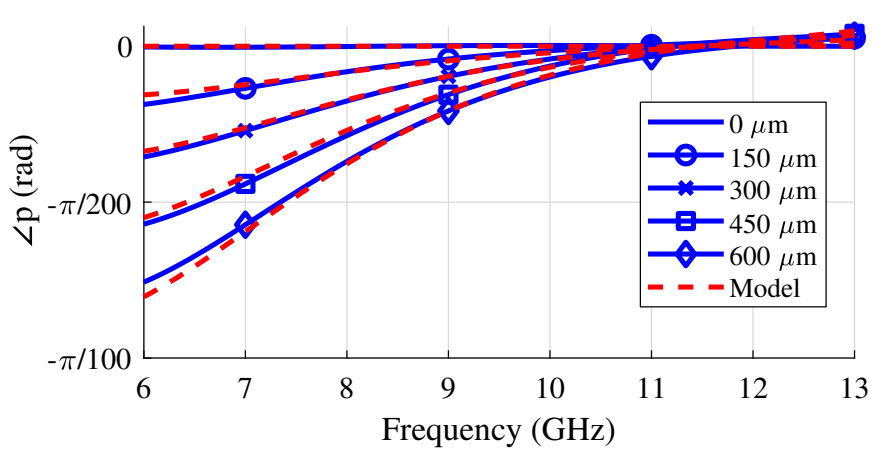

(b)

Fig. 8. Simulated values of $p$ of the SFCP unit cell for several asymmetries versus those computed by the model. (a) Magnitude. (b) Phase.

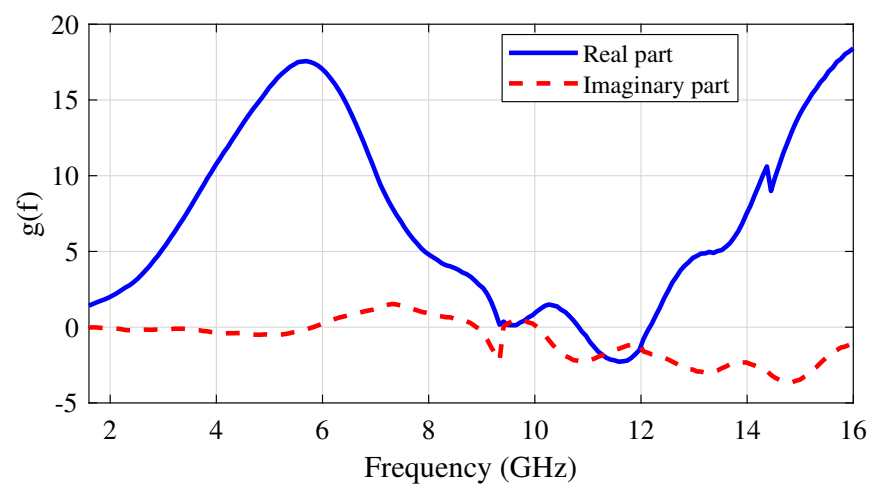

Fig. 9. $g(f)$ function of the asymmetry model for the complementary-stripslot unit cell.

handle two-ports with isolated ports $\left(y_{12}=0\right.$ or $\left.z_{12}=0\right)$. Explicit and simple instructions for the straightforward extraction of the equivalent circuit element values are provided.

The equivalent circuit has been validated by analyzing two different structures. In both cases, the results have proved and illustrated its capabilities and robustness against numerical and measurement uncertainties in a very broad band. An important feature of the proposed equivalent circuit is that it is capable of displaying the structure asymmetry explicitly. As a consequence, it is most convenient when the two-port has two identifiable eigenstates, since it easily models the underlying physics of the structure. This capability has been illustrated by

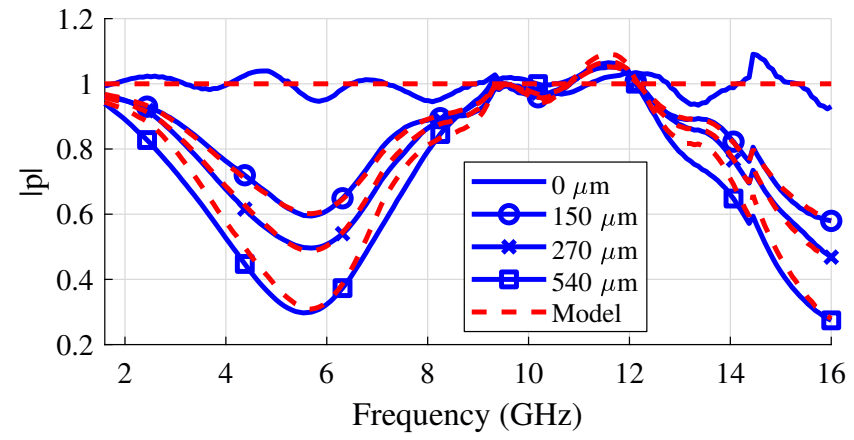

(a)

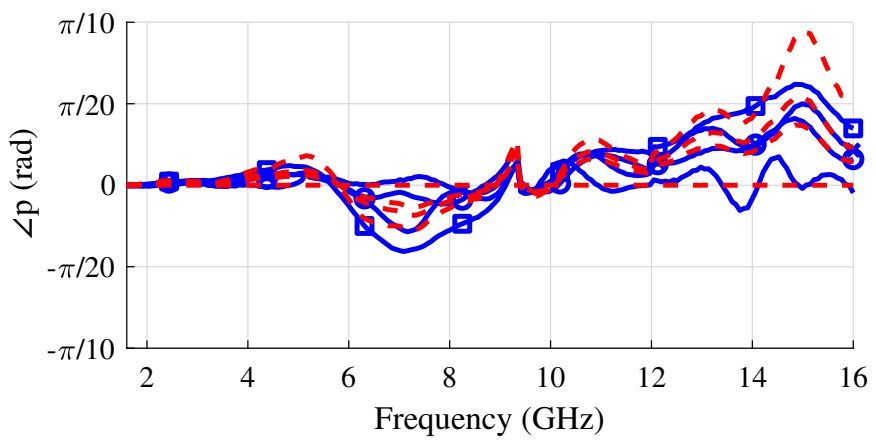

(b)

Fig. 10. Simulated values of $p$ of the complementary-strip-slot unit cell for several asymmetries versus those computed by the model. (a) Magnitude. (b) Phase.

both cases; the turns ratio practically absorbs the asymmetry of the network leaving the immittances almost unperturbed. When this happens, it is possible to model the turns ratio with a simple expression in a very wide band as a function of the asymmetry. The model is able to accurately replicate the parameters of the structure when the asymmetry level is not too high. This feature allows the design of the asymmetry in unit cells to maximize the mitigation of the stopband in LWAs, but could be easily applied to other electromagnetic structures in which slight asymmetries play an important role.

The excellent simplicity, eigenstate-based derivation and extraordinary behavior in modeling structures of different nature makes the proposed equivalent circuit a good candidate for modeling lossy non-symmetric reciprocal two-ports, especially when physical insight is pursued. Indeed, the highlighted capabilities of the proposed network suggest that it can be regarded as a generalization of the symmetric lattice network for lossy non-symmetric two-ports.

\section{APPENDIX}

Let $[A]$ be a square matrix with complex elements and let $\pi\{[A]\}, \nu\{[A]\}$ and $\delta\{[A]\}$ be the number of eigenvalues of $[A]$ with positive real part, negative real part and zero real part, respectively. The triple pair $(\pi\{[A]\}, \nu\{[A]\}, \delta\{[A]\})$ is called the inertia of $[A]$. According to [17], there exists a Hermitian matrix $[H]$ so that the matrix $[A][H]+[H][A]^{*}$ is 


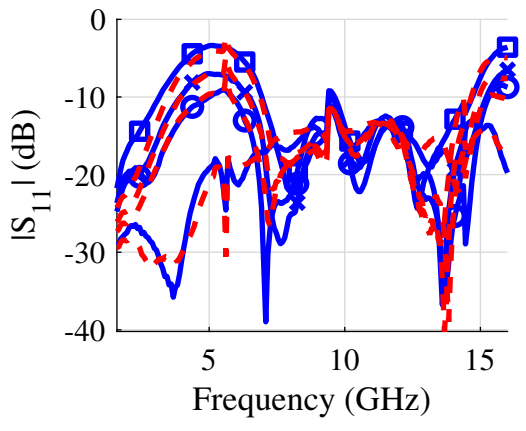

(a)

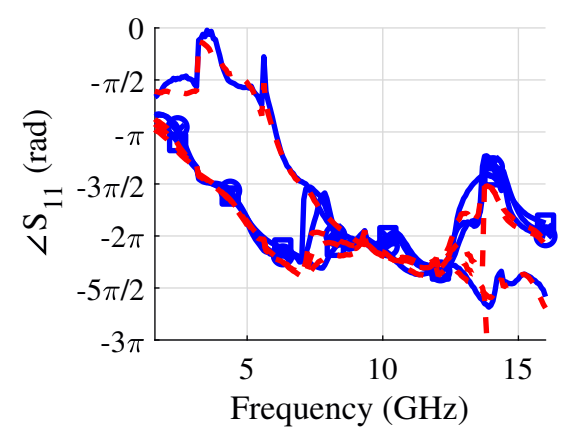

(d)

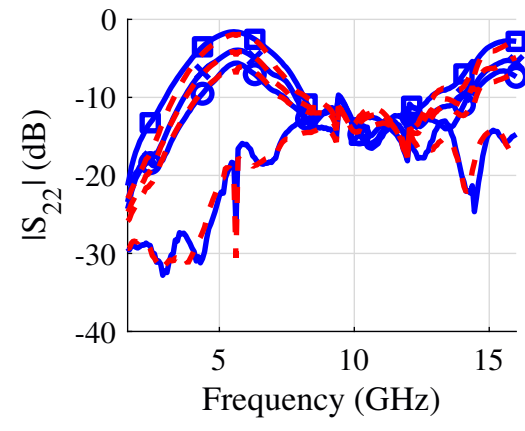

(b)

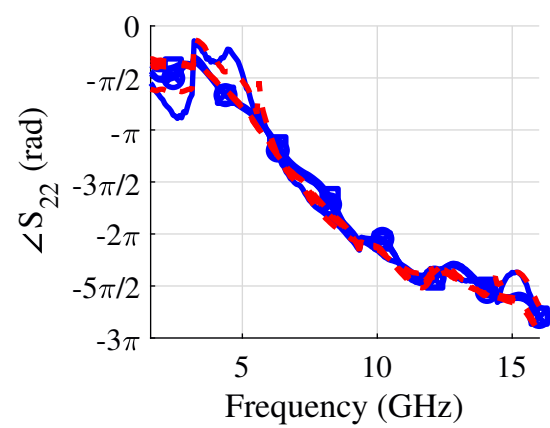

(e)

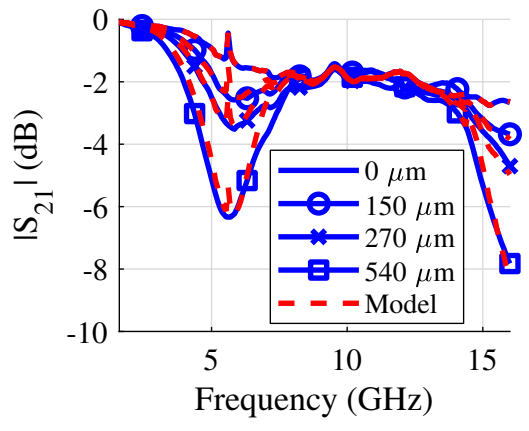

(c)

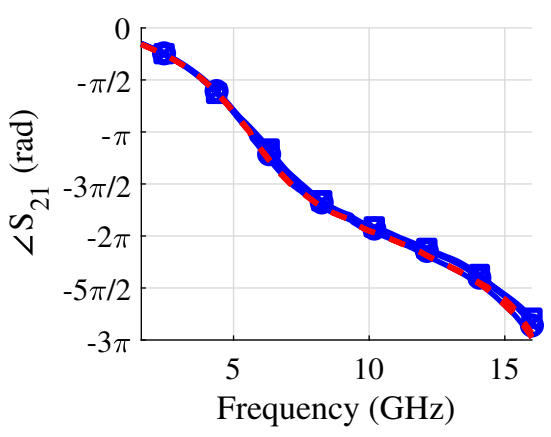

(f)

Fig. 11. S parameters of the measured strip-slot unit cells for several asymmetries versus those computed by the model. (a) Magnitude of $S_{11}$. (b) Magnitude of $S_{22}$. (c) Magnitude of $S_{21}$. (d) Phase of $S_{11}$. (e) Phase of $S_{22}$. (f) Phase of $S_{21}$.

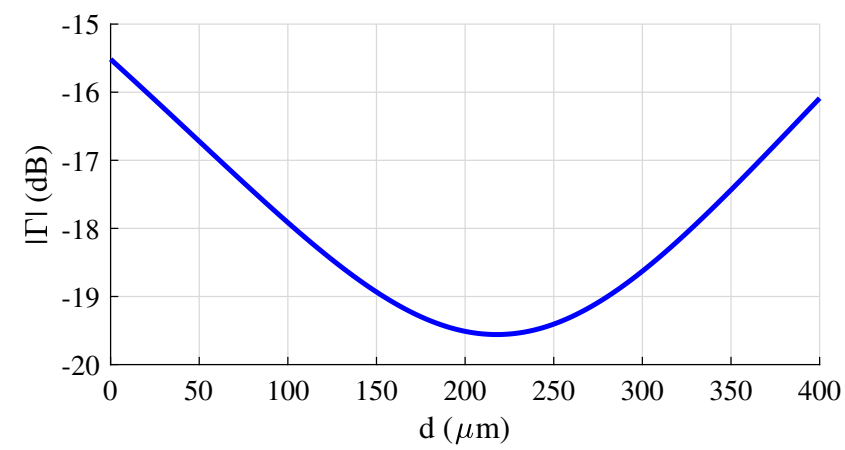

Fig. 12. Computed reflection coefficient of the SFCP unit cell in function of the asymmetry level using the proposed model.

positive definite if and only if $\delta=0$. In this case, $[A]$ and $[H]$ have the same inertia.

Let us apply this theorem to the admittance matrix of a passive and reciprocal circuit. First, it is possible to define the matrix formed by the real parts of the elements of $[Y]$ as

$$
\operatorname{Re}\{[Y]\}=\frac{[Y]+[Y]^{*}}{2}=\left[\begin{array}{ll}
r_{11} & r_{12} \\
r_{12} & r_{22}
\end{array}\right] .
$$

According to the energy theorem, the components of the admittance matrix must fulfill the following conditions [18]:

$$
\begin{aligned}
& r_{11} \geq 0 \\
& r_{22} \geq 0
\end{aligned}
$$

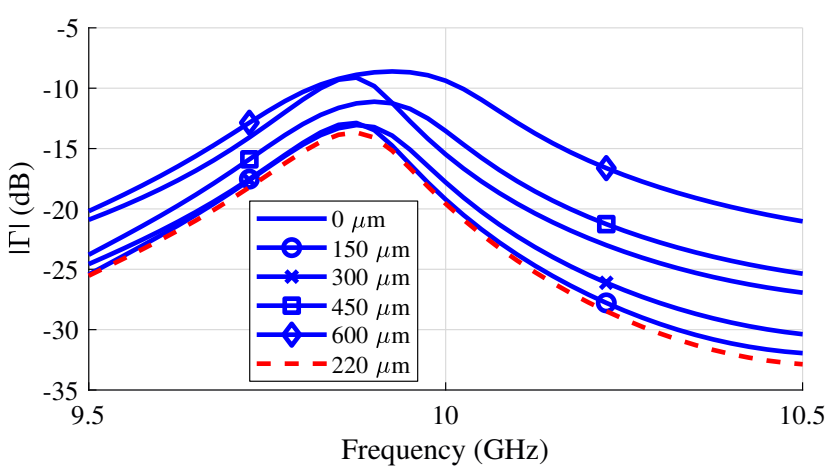

Fig. 13. Simulated reflection coefficient of the SFCP unit cell for different asymmetry levels.

$$
r_{11} r_{22}-r_{12}^{2} \geq 0 \text {. }
$$

Since the circuit is reciprocal, $\operatorname{Re}\{[Y]\}$ is a symmetric matrix and, thus, its eigenvalues are real. Furthermore, if the circuit is lossy, $r_{11}>0$ and $r_{22}>0$, and then, by using the properties of the eigenvalues,

$$
\begin{aligned}
& \operatorname{tr}\left\{\left[\begin{array}{ll}
r_{11} & r_{12} \\
r_{12} & r_{22}
\end{array}\right]\right\}=r_{11}+r_{22}=\lambda_{r 1}+\lambda_{r 2}>0 \\
& \operatorname{det}\left\{\left[\begin{array}{ll}
r_{11} & r_{12} \\
r_{12} & r_{22}
\end{array}\right]\right\}=r_{11} r_{22}-r_{12}^{2}=\lambda_{r 1} \lambda_{r 2}>0,
\end{aligned}
$$

which implies that the $\operatorname{Re}\{[Y]\}$ matrix is definite positive if the circuit is reciprocal and lossy, since its eigenvalues, $\lambda_{r 1}$ and $\lambda_{r 2}$, are real and positive. 
It is possible to write (15) as

$$
\operatorname{Re}\{[Y]\}=\frac{1}{2}\left([Y][I]+[I][Y]^{*}\right)
$$

where $[I]$ denotes the identity matrix, which is Hermitian, and so, it is possible to apply the theorem from [17]. This means that the inertia of $[Y]$ and $[I]$ are the same. The eigenvalues of $[I]$ are $\lambda_{I 1}=\lambda_{I 2}=1$, real and positive, then,

$$
\pi\{[I]\}=2 \Rightarrow \pi\{[Y]\}=2 .
$$

This way, it is proved that the real parts of the eigenvalues of the $[Y]$ matrix are always positive if the circuit is lossy and reciprocal.

If the circuit is lossless, it is straightforward to prove that the eigenvalues of $[Y]$ are purely imaginary numbers. The admittance parameters of a lossless circuit are always purely imaginary and, thus, it is possible to define a symmetric and real matrix $\operatorname{Im}\{[Y]\}$ as

$$
\operatorname{Im}\{[Y]\}=\left[\begin{array}{ll}
i_{11} & i_{12} \\
i_{12} & i_{22}
\end{array}\right]=-j[Y]
$$

where $i_{i j}$ are the imaginary parts of the circuit admittance parameters. Let us write the eigenstate decomposition as a function of this matrix:

$$
j\left[\begin{array}{ll}
i_{11} & i_{12} \\
i_{12} & i_{22}
\end{array}\right] \overrightarrow{v_{i}}=j \lambda_{i} \overrightarrow{v_{i}}
$$

where $\lambda_{i}$ is the eigenvalue of $\operatorname{Im}\{[Y]\}$ and $\overrightarrow{v_{i}}$ the eigenvector of the $[Y]$ matrix. Since the $\operatorname{Im}\{[Y]\}$ matrix is real and symmetric, its eigenvalues are real and, consequently, the eigenvalues of $[Y]$ are purely imaginary numbers.

\section{REFERENCES}

[1] S. Otto, A. Al-Bassam, A. Rennings, K. Solbach, and C. Caloz, "Radiation efficiency of longitudinally symmetric and asymmetric periodic leaky-wave antennas," IEEE Antennas Wirel. Propag. Lett., vol. 11, pp. 612-615, Jun. 2012.

[2] — , "Transversal asymmetry in periodic leaky-wave antennas for Bloch impedance and radiation efficiency equalization through broadside," IEEE Trans. Antennas Propag., vol. 62, no. 10, pp. 5037-5054, Oct. 2014.

[3] F. Mesa, R. Rodriguez-Berral, and F. Medina, "Unlocking complexity using the ECA: The equivalent circuit model as an efficient and physically insightful tool for microwave engineering," IEEE Microw. Mag., vol. 19, no. 4, pp. 44-65, Jun. 2018.

[4] - "On the computation of the dispersion diagram of symmetric onedimensionally periodic structures," Symmetry, vol. 10, p. 307, Aug. 2018.

[5] C. G. Montgomery, R. H. Dicke, and E. M. Purcell, Principles of Microwave Circuits. MIT Radiation Lab series, 1948.

[6] N. Marcuvitz, Waveguide Handbook, ser. IEE electromagnetic waves series. The Institution of Engineering and Technology, 1986.

[7] L. B. Felsen and A. A. Oliner, "Determination of equivalent circuit parameters for dissipative microwave structures," in Proc. IRE, vol. 42, no. 2, 1954, pp. 477-483.

[8] A. Weissfloch, Elek. Nach. Tech. vol. 19, 1942.

[9] L. Zappelli, "Equivalent circuits of lossy two-port waveguide devices," IEEE Trans. Microw. Theory Techn., vol. 67, no. 10, pp. 4095-4106, Oct. 2019.

[10] E. Abdo-Sánchez, C. Camacho-Peñalosa, T. M. Martín-Guerrero, and J. Esteban, "Equivalent circuits for nonsymmetric reciprocal two ports based on eigenstate formulation," IEEE Trans. Microw. Theory Techn., vol. 65, no. 12, pp. 4812-4822, Dec. 2017.

[11] S. Wane and D. Bajon, "Broadband equivalent circuit derivation for multi-port circuits based on eigen-state formulation," in IEEE MTT-S Int. Micro. Symp. Dig., 2009, pp. 305-308.
[12] J. Lu, H. J. Ng, D. Kissinger, C. F. Jou, and L. Wu, "Design of a novel microstrip Franklin leaky-wave antenna using the eigenstate approach," IEEE Trans. Antennas Propag., vol. 67, no. 7, pp. 4484-4494, Jul. 2019.

[13] L. P. Huelsman, Circuits, Matrices and Linear Vector Spaces, ser. Dover Books on Electrical Engineering. Dover Publications, 2013.

[14] E. Abdo-Sánchez, T. Martín-Guerrero, J. Esteban, and C. CamachoPeñalosa, "Short dual-band planar leaky-wave antenna with broadside effect mitigation," IET Microw. Antennas Propag., vol. 10, no. 5, pp. 574-578, Apr. 2016.

[15] E. Abdo-Sánchez, J. E. Page, T. M. Martín-Guerrero, J. Esteban, and C. Camacho-Peñalosa, "Planar broadband slot radiating element based on microstrip-slot coupling for series-fed arrays," IEEE Trans. Antennas Propag., vol. 60, no. 12, pp. 6037-6042, Dec. 2012.

[16] A. Hessel, "Travelling-wave antennas," in Antenna Theory, R. E. Collin and F. J. Zucker, Eds. New York: McGraw-Hill, 1969, ch. 19.

[17] A. Ostrowski and H. Schneider, "Some theorems on the inertia of general matrices," J. Math. Anal. Appl., vol. 4, no. 1, pp. 72 - 84, Feb. 1962.

[18] M. E. VanValkenburg, Introduction to Modern Network Synthesis. Wiley, 1960. 